\title{
Inhibitory effect of icariin on osteosarcoma cell proliferation via the Wnt/ $\beta$-catenin signaling pathway
}

\author{
YUXIN REN $^{1 *}$, FUQIANG ZHU ${ }^{1 *}$ and ZHENDONG LIU ${ }^{2}$ \\ Departments of ${ }^{1}$ Spinal Surgery and ${ }^{2}$ Orthopedics, Dezhou People's Hospital, Dezhou, Shandong 253014, P.R. China
}

Received July 20, 2017; Accepted November 17, 2017

DOI: $10.3892 / \mathrm{ol} .2018 .8821$

\begin{abstract}
Osteosarcoma, is a kind of malignant tumor derived from malignant interstitial cells. The pathogenesis of osteosarcoma remains unclear and despite use of chemotherapy drugs, resistance to these drugs affects the success of treatment. The present study was conducted to investigate the effects of icariin (ICA) on osteosarcoma cell proliferation and to investigate the role of the $\mathrm{Wnt} / \beta$-catenin signaling pathway in the inhibition process of ICA on osteosarcoma cell proliferation. Different concentrations of ICA were selected to treat the osteosarcoma cell line $143 \mathrm{~B}$ for $24 \mathrm{~h}$, and then the onset concentration of ICA when it inhibited the growth of osteosarcoma cancer cell line 143B was detected via an MTT assay. The effect of ICA on the apoptosis of colon cancer cell line 143B under this concentration was detected using a flow cytometer. RNA in osteosarcoma cell line 143B was extracted, followed by reverse transcription. The expression levels of related and apoptotic proteins in the $\mathrm{Wnt} / \beta$-catenin signaling pathway using ICA were detected by semiquantitative PCR and western blot analysis, respectively. The expression quantities of vascular endothelial growth factor (VEGF) and MMP-9 were detected by ELISA. MTT assay showed that ICA inhibited the growth of 143B when its concentration was $5 \mu \mathrm{M}(\mathrm{p}<0.01)$. Flow cytometry showed that the number of apoptotic cells after ICA treatment was significantly higher than that in control group $(\mathrm{p}<0.01)$. RNA in osteosarcoma cell line 143B was extracted, followed by reverse transcription. Semi-quantitative PCR and western blot analysis revealed that the expression levels of p-GSK3 $\beta$, $\beta$-catenin, c-Myc and cyclin D1 in cells after ICA treatment were significantly downregulated $(p<0.01)$, while the expression level of caspase- 3 was significantly increased $(\mathrm{p}<0.01)$.
\end{abstract}

Correspondence to: Dr Zhendong Liu, Department of Orthopedics, Dezhou People's Hospital, 1751 Xinhu Street, Dezhou, Shandong 253014, P.R. China

E-mail: liuzhd123@126.com

*Contributed equally

Key words: icariin, osteosarcoma, Wnt/ $\beta$-catenin signaling pathway, proliferation
ELISA showed that the expression quantities of VEGF and MMP-9 were significantly decreased $(\mathrm{p}<0.01)$. Thus, ICA can significantly inhibit osteosarcoma cell proliferation and promote osteosarcoma cell apoptosis, which may be realized by affecting the expression of the Wnt/ $\beta$-catenin signaling pathway and blocking the expression of related proteins.

\section{Introduction}

Osteosarcoma, as a kind of malignant tumor derived from malignant interstitial cells, has certain osteoid characteristics, including a strong migration capacity and frequent systemic metastasis (1). The pathogenesis of osteosarcoma remains unclear and the osteosarcoma of osteoblasts is a clinically common form at present, which generally occurs in the metaphysis of long-tubular bone, but seldom in the axial skeleton $(2,3)$. The malignant grade of osteosarcoma is high, micro-lesion metastasis is possible during diagnosis, while lung tissue is a common metastatic site (4). Prior to the application of chemotherapeutic drugs, amputation was commonly used in the treatment of osteosarcoma. The rise and development of chemotherapy drugs has been useful in the treatment of osteosarcoma. However, drug resistance of chemotherapy drugs delays the treatment of osteosarcoma (5). Icariin (ICA), also known as Epimedium brevicornu, is sweet and warm. It has the effect of nourishing kidney and strengthening yang, strengthening tendons and bones, dispelling wind and eliminating dampness, expelling furuncle and resolving carbuncle. ICA is often clinically used in the treatment of flaccid bones and muscles and rheumatic arthralgia (6-8). Recent findings have shown that ICA has the effect of promoting osteoblast proliferation and increasing ALP activity in osteoblasts, thus promoting the calcification of bone matrix and enhancing bone strength (9). Zhang et al (10) found that ICA can significantly increase osteocytes and promote osteocyte differentiation. Zhang et al (11) found that ICA can increase the apoptosis of osteoclastoma. However, to the best of our knowledge, there is no report currently available on the effect of ICA on osteosarcoma cells and its mechanism.

In this study, the effect of ICA on osteosarcoma cell proliferation and its mechanism were studied to clarify the mechanism of ICA for osteosarcoma cells, in order to provide new ideas for the efficacious development of ICA and clinical treatment of osteosarcoma. 


\section{Materials and methods}

Instruments and materials. Osteosarcoma cancer cell line 143B (Cell Bank of the Chinese Academy of Sciences, Shanghai, China), methyl thiazolyl tetrazolium (MTT) and DMSO (both from Sigma-Aldrich; Merck KGaA, Darmstadt, Germany), ICA (Aladdin Shanghai Biochemical Technology Co., Ltd., Shanghai, China), TRIzol kit and reverse transcription kit (both from Invitrogen, Carlsbad, CA, USA), VEGA ELISA kit and MMP-9 ELISA kit (both from R\&D Systems, Inc., Minneapolis, MN, USA), rabbit anti-DKK1, rabbit anticaspase-3, rabbit anti- $\beta$-catenin, rabbit anti-GSK3 $\beta$, rabbit anti-pGSK3 $\beta$ (Ser9) and GAPDH (all from Cell Signaling Technology, Inc., Danvers, MA, USA), ECI luminescent solution (Invitrogen), inversed fluorescent microscope (Thermo Fisher Scientific GmbH, Dreieich, Germany), cell culture flask (Corning Inc., Corning, NY, USA), pipettor (Eppendorf, Hamburg, Germany), PCR instrument (Applied Biosystems, Foster City, CA, USA), UV imaging system (Biometra, Goettingen, Germany), and electronic balance (BP121S; Sartorius AG, Goettingen, Germany) were used in the study. Any other equipment and reagents were previously described in the relevant section.

Detection of inhibitory effect of ICA on osteosarcoma cell growth via MTT assay. After osteosarcoma cancer cell line 143B was revived (12), it was placed in the incubator, and cells in the logarithmic growth phase were inoculated onto the 96 -well plate after subculture $\left(8 \times 10^{3} /\right.$ well). [Of note, the 143B cell line used in the present study is possibly identified as osteosarcoma cell line HTK-osteosarcoma cell line (13); however, it did not affect the conclusions drawn in the study.] After inoculation for $24 \mathrm{~h}$, the serum was deprived for $24 \mathrm{~h}$ and ICA was added at different concentrations (20, 10, 5, 2, 1 and $0.1 \mu \mathrm{M})$. The blank control group and the positive drug group (cisplatin) were then set up. After incubation with medication for $24 \mathrm{~h}, 1 \%$ MTT was added to incubate the cells for $4 \mathrm{~h}$ in the dark. The medium was discarded and $150 \mu \mathrm{l}$ dimethyl sulfoxide was added to each well. After vibration for $10 \mathrm{~min}$, the absorbance value at $570 \mathrm{~nm}$ was measured using the microplate reader (Thermo Fisher Scientific; Waltham, MA, USA).

Effects of ICA on osteosarcoma cell apoptosis. The 143B colon cancer cell line was cultured as follows. The cell density was adjusted to $5 \times 10^{6}$ cells $/ \mathrm{ml}$ and added into the 6-well plate, and the cells were then divided into the blank control, ICA $(5 \mu \mathrm{M})$ and positive drug groups. After treatment for $24 \mathrm{~h}$, the cells were washed with pre-cooled PBS twice, re-suspended and centrifuged at 12,000 $\mathrm{x}$ g for $5 \mathrm{~min}$. Fluorescent solution was added to incubate the cells at room temperature for $15 \mathrm{~min}$ in the dark. The cells were then detected using a flow cytometer (Thermo Fisher Scientific, Inc., Waltham, MA, USA).

Detection of the expression of related genes via semi-quantitative PCR. The cell proteins treated with $5 \mu \mathrm{M}$ ICA for $24 \mathrm{~h}$ were extracted using a TRIzol kit, and the supernatant was discarded via centrifugation for $10 \mathrm{~min}$ at $6,000 \mathrm{x}$ g. The RNA integrity was confirmed by agarose gel electrophoresis.
Table I. PCR primer.

\begin{tabular}{ll}
\hline Gene name & \multicolumn{1}{c}{ Primer sequences } \\
\hline c-Myc & F: 5'-ATCCAGAGACAAGACATGTAC-3' \\
& R:5'-TTCAGATGTTCTAAGCCTACGG-3' \\
$\beta$-catenin & F: 5'-TGGCGGTTTGCGGTGGAC-3' \\
& R: 5'-CCAGTGCAGGGTCCGAGGT-3' \\
Cyclin D1 & F: 5'-GATGATTGGCATGGCTTT-3' \\
& R: 5'-CACCTTCCGTTCCAGTTT-3' \\
Caspase-3 & F: 5'-GATGGGACTGTGGTTACCGT-3' \\
& R: 5'-GGTGAAACTCTTGCCTCGTC-3' \\
MMP-9 & F: 5'-GATGATTGGCATGGCTTT-3' \\
& R: 5'-GCCATACGCTGACCTTTCA-3' \\
VEGF & F: 5'-GGAGTCCATTGGTGCTTGA-3' \\
RAPDH & R: 5'-ACACCCTTCCAATGTGCC-3' \\
& R: 5'-CACGATTGGCATGGCTTT-3'
\end{tabular}

F, forward; R, reverse; VEGF, vascular endothelial growth factor.

The results of electrophoresis showed that $28 \mathrm{~S}, 18 \mathrm{~S}$ and $5 \mathrm{~S}$ bands were clear, and the brightness of $28 \mathrm{~S}$ band was approximately twice of that of $18 \mathrm{~S}$ band, indicating that RNA was intact and can be used for subsequent experiments. cDNA was obtained via reverse transcription using the reverse transcription kit. An appropriate internal reference was selected. The expression levels of c-Myc, cyclin D1 and $\beta$-catenin in the $\mathrm{Wnt} / \beta$-catenin signaling pathway, apoptosis-related gene caspase- 3 and migration-related gene MMP-9 and VEGF were detected via semi-quantitative PCR. Reaction conditions were: denaturation, $95^{\circ} \mathrm{C}$ for $30 \mathrm{sec}$; annealing, $64^{\circ} \mathrm{C}$ for $25 \mathrm{sec}$; and extension $72^{\circ} \mathrm{C}$ for $30 \mathrm{sec}$, for a total of 35 cycles. Primers were produced by Tiangen Biotech Co., Ltd. (Beijing, China). Primer sequences are shown in Table I. After the reaction, agarose gel electrophoresis was performed, followed by observation using a UV imaging system.

Detection of the expression of related proteins via western blot analysis. The cells in the logarithmic growth phase were inoculated onto the 96-well plate, and the blank control and ICA groups $(5 \mu \mathrm{M})$ were established. After medication for $24 \mathrm{~h}$, the protein was extracted and quantified, followed by separation via SDS-PAGE and the protein was transferred to PVDF membrane. The target band was cut off after sealing for $2 \mathrm{~h}$. The target protein antibody [anti-caspase-3, rabbit monoclonal antibody, catalogue no. 9665 ; anti- $\beta$-catenin, rabbit monoclonal antibody, catalogue no. 9582; monoclonal antibody anti-GSK3 $\beta$, monoclonal antibody, rabbit 12456; anti-pGSK3 $\beta$ (Ser9), monoclonal antibody, rabbit 5558; cyclin D1, rabbit monoclonal antibody, catalogue no. 2978; and CMYc, rabbit monoclonal antibody, catalogue no.13987 were procured from Cell Signaling Technology, Inc., Danvers, MA, USA] was used to incubate the protein at $4^{\circ} \mathrm{C}$ overnight. After washing with Tris-buffered saline Tween-20 (TBST) three times ( $5 \mathrm{~min} /$ time), the secondary antibody was incubated at room temperature for $2 \mathrm{~h}$. After washing with TBST 


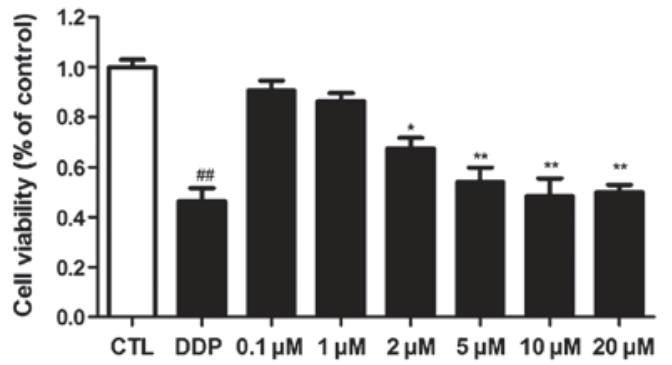

Figure 1. Detection of inhibitory effect of ICA on the growth of osteosarcoma cell 143B via MTT assay. When the ICA concentration reached $5 \mu \mathrm{M}$, the survival rate of $143 \mathrm{~B}$ was significantly decreased $\left({ }^{* *} \mathrm{p}<0.05\right.$ and $\left.^{\# \#} \mathrm{p}<0.01\right)$, and this concentration was used as the concentration in subsequent experiments. ICA, icariin. ${ }^{*} \mathrm{P}>0.05$.

A

\section{CTL group}

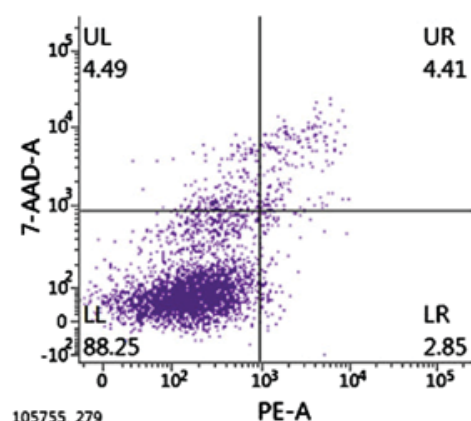

B

ICA (5 $\mu \mathrm{M})$ group

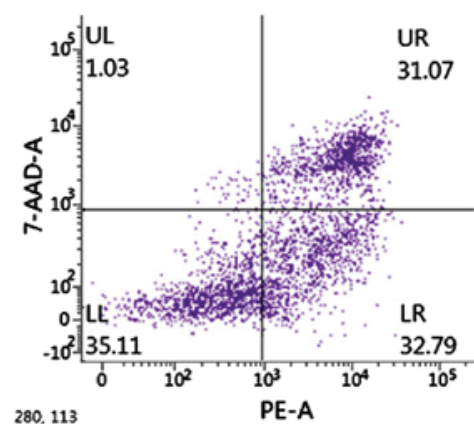

Figure 2. Detection of cell apoptosis via flow cytometer. (A) Blank control group, and (B) ICA (5 $\mu \mathrm{M})$ group. Compared with the blank control group, $5 \mu \mathrm{M}$ ICA significantly increased the apoptosis of osteosarcoma cell 143B, and the difference was statistically significant. ICA, icariin.

again three times, the appropriate amount of ECL solution (solution $\mathrm{A}$ and $\mathrm{B}$ were mixed at a ratio of 1:1) was added in the dark, followed by performing of the experiment, and the performing time was determined according to the fluorescence intensity of the protein band. Following development and fixation, the band was scanned and received gray value analysis via ImageJ software. The expression levels of related proteins in the Wnt/ $\beta$-catenin signaling pathway, p-GSK3 $\beta$, c-Myc, cyclin $\mathrm{D} 1$ and $\beta$-catenin, and apoptosis protein caspase-3 were detected.

Detection of the expression levels of VEGF and MMP-9 via ELISA. The cells in the logarithmic growth phase were inoculated onto the 24-well plate, and the blank control and
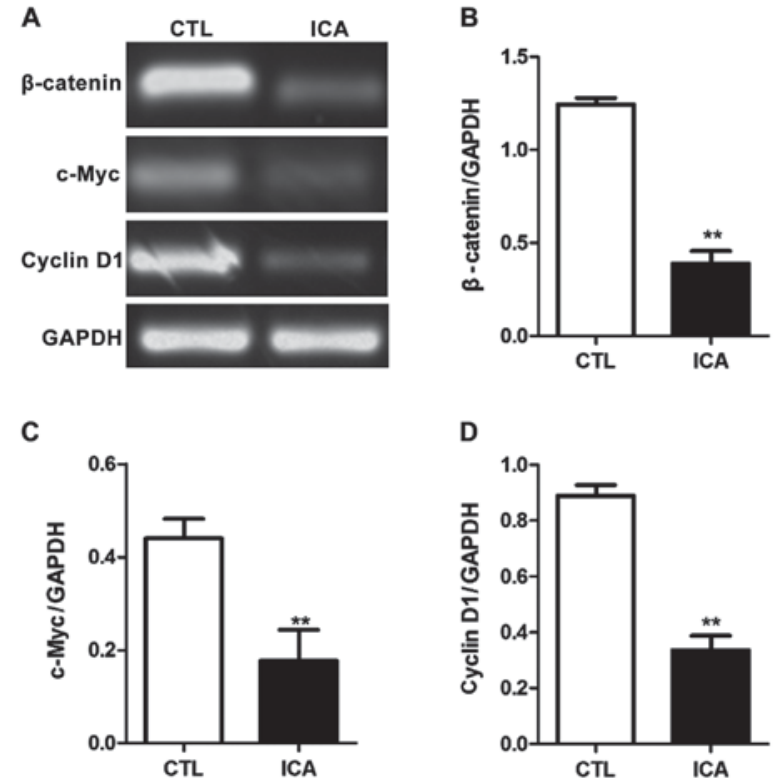

Figure 3. Detection of effect of ICA on the expression levels of related genes in the Wnt/ $\beta$-catenin signaling pathway via semi-quantitative PCR. (A) Agarose gel electrophoresis. (B-D) Statistical charts of the relative expression levels of $\beta$-catenin, $\mathrm{c}-\mathrm{Myc}$ and cyclin D1. mRNA expression levels in $\beta$-catenin, c-Myc and cyclin D1 in osteosarcoma cells were significantly decreased after ICA treatment $\left.{ }^{* * *} \mathrm{p}<0.01\right)$. ICA, icariin.
A

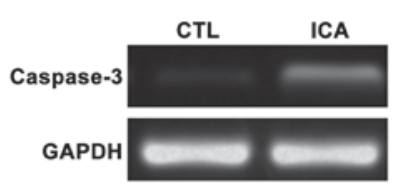

B

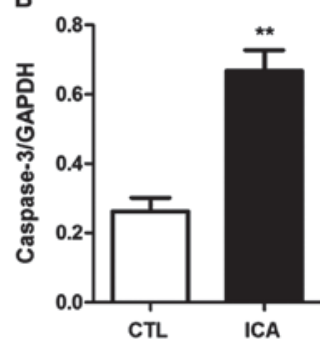

Figure 4. Detection of the effect of ICA on the mRNA expression level in caspase-3 via semi-quantitative PCR. (A) Chart of agarose gel electrophoresis (B) Statistical chart of relative expression level. After ICA treatment, the mRNA expression level in caspase-3 in osteosarcoma cells was significantly increased $(* * \mathrm{p}<0.01)$. ICA, icariin

ICA groups $(5 \mu \mathrm{M})$ were set up. After medication for $24 \mathrm{~h}$, the supernatant was collected and the expression levels of VEGF and MMP-9 in cells were detected using an ELISA kit.

Statistical analysis. Data were presented as mean \pm standard deviation and analyzed by SPSS 19.0 (SPSS Inc., Chicago, IL, USA) software. The t-test was used for measurement data, while the Chi-square test was used for enumeration data. One-way analysis of variance (ANOVA) was used for other data. Bonferronic method was used for pairwise comparison under homogeneity of variance, while the Welch method was used under heterogeneity of variance. Dunnett's T3 method was used for multiple comparisons.

\section{Results}

Inhibitory effect of ICA on osteosarcoma cell growth. The inhibitory effect of ICA on the osteosarcoma cell 143B was 
A

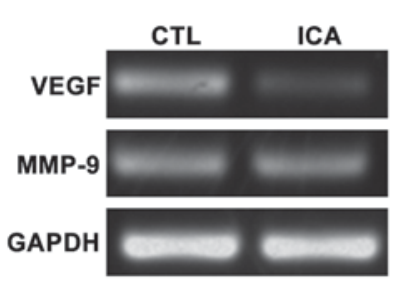

B

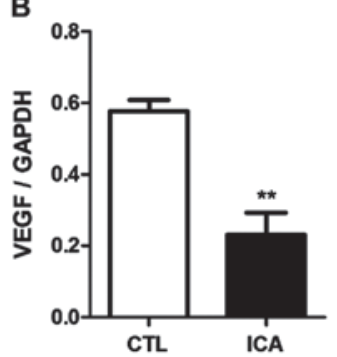

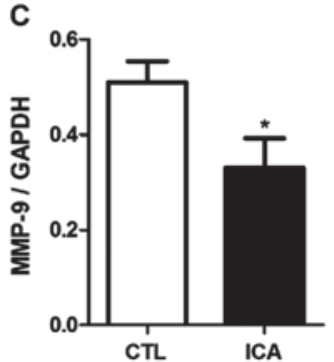

Figure 5. Detection of effect of ICA on mRNA expression levels in MMP-9 and VEGF via semi-quantitative PCR. (A) Chart of agarose gel electrophoresis. (B and C) Statistical charts of relative expression levels. After ICA treatment, mRNA expression levels in MMP-9 and VEGF in osteosarcoma cells were significantly decreased $\left(* \mathrm{p}<0.05,{ }^{* *} \mathrm{p}<0.01\right)$. ICA, icariin.
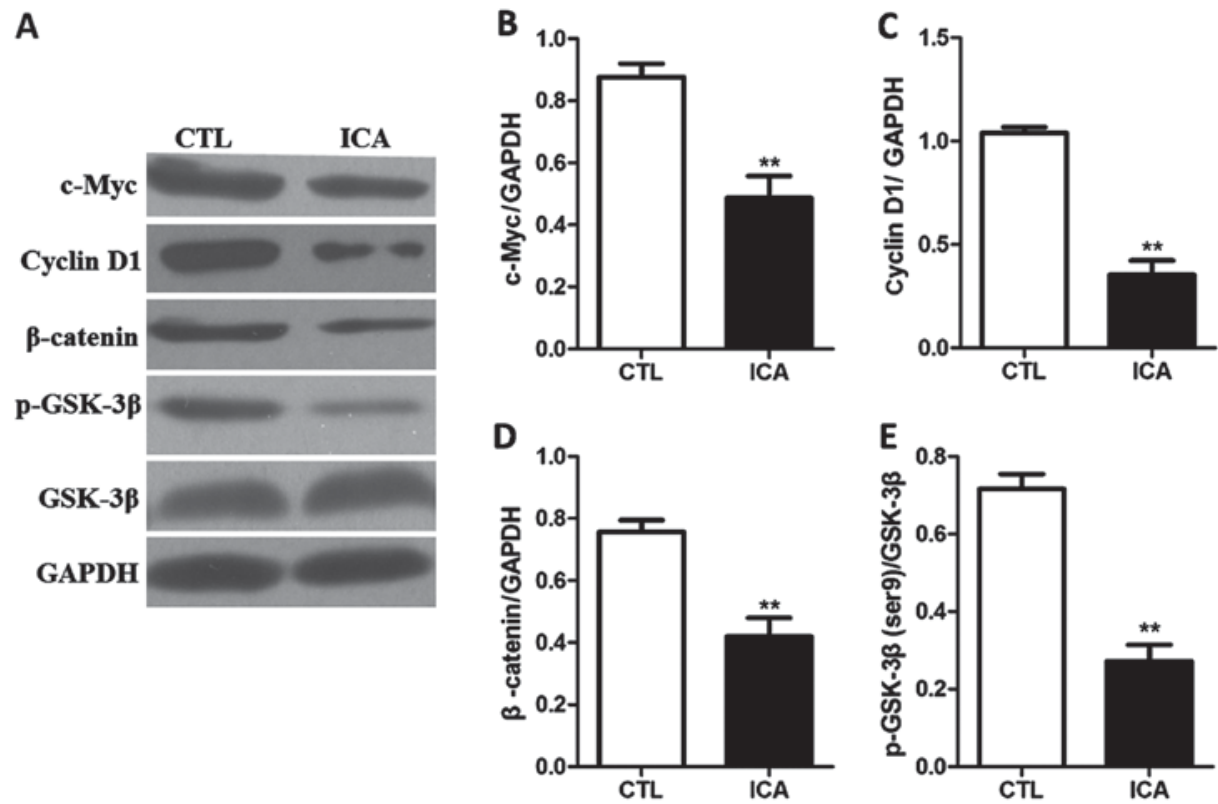

Figure 6. Detection of effect of ICA on expression levels of related proteins in the Wnt/ $\beta$-catenin signaling pathway via western blot analysis. (A) Western blot bands. (B-E) Statistical charts of $\beta$-catenin, p-GSK3 $\beta$, c-Myc and cyclin D1. The results show that the protein expression levels in $\beta$-catenin, p-GSK3 $\beta$, c-Myc and cyclin D1 were significantly decreased after ICA treatment $\left({ }^{* *} \mathrm{p}<0.01\right)$. ICA, icariin.
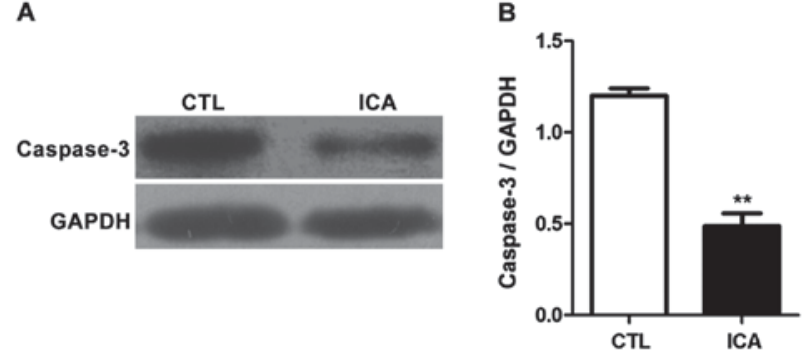

Figure 7. Detection of effect of ICA on the protein expression level in caspase-3 via western blot analysis. (A) Western blot bands. (B) Statistical chart. The results showed that the protein expression level in caspase-3 in osteosarcoma cells was significantly increased after ICA treatment $(* * 0<0.01)$. ICA, icariin.

detected via MTT assay. The results are shown in Fig. I. ICA concentrations were set as $20,10,5,2,1$ and $0.1 \mu \mathrm{M}$, and the blank control and positive control groups (cisplatin) were established. The results showed that ICA significantly inhibited the 143B cell growth when its concentration reached $5 \mu \mathrm{M}(\mathrm{p}<0.01)$.
A
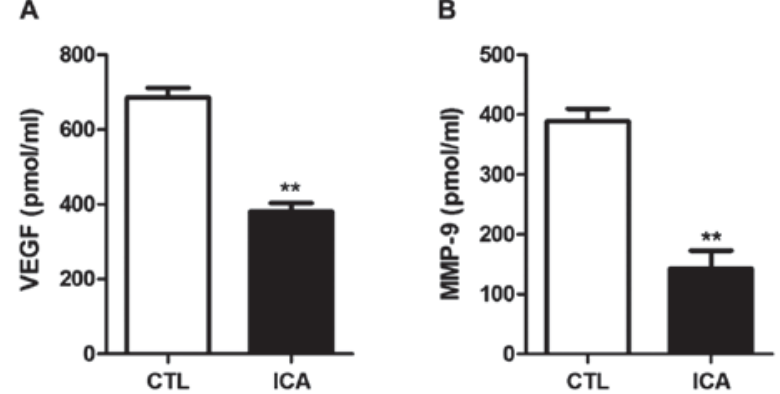

Figure 8. Detection of the expression levels of VEGF and MMP-9 via ELISA Statistical chart of (A) VEGF and (B) MMP-9 expression level. The results show that the expression levels of VEGF and MMP-9 in osteosarcoma cells were significantly decreased after ICA treatment $\left({ }^{* *} \mathrm{p}<0.01\right)$. ICA, icariin

Effects of ICA on osteosarcoma cell apoptosis. The effects of ICA on the apoptosis of osteosarcoma cell 143B were detected via a flow cytometer. The results are shown in Fig. 2. After $5 \mu \mathrm{M}$ ICA was added to the osteosarcoma cell $143 \mathrm{~B}$, the number of apoptotic cells in the ICA group was significantly 
increased compared with that in the blank control group, and the difference was statistically significant $(\mathrm{p}<0.01)$.

Detection of related gene expressions via semi-quantitative $P C R$. After the RNA of osteosarcoma cell 143B treated with ICA was extracted, the relative expression quantities of related genes were detected via semi-quantitative PCR with GAPDH as the internal reference. The results are shown in Figs. 3-5. Fig. 3 shows that compared with those in the blank control group, the expression levels of $\beta$-catenin were decreased after ICA treatment, and the expression levels of its downstream genes c-Myc and cyclin D1 were also significantly decreased $(\mathrm{p}<0.01)$. Fig. 4 shows that the expression level of caspase-3 was significantly increased after ICA treatment $(\mathrm{p}<0.01)$. Fig. 5 shows that the expression levels of MMP-9 and VEGF were decreased after ICA treatment $(\mathrm{p}<0.01)$.

Detection of expression levels of related proteins via western blot analysis. The expression levels of related proteins in osteosarcoma cell $143 \mathrm{~B}$ treated with ICA $(5 \mu \mathrm{M})$ were detected via western blot analysis, and the results are shown in Figs. 6 and 7. Fig. 6 shows that the protein expression levels in p-GSK3 $\beta$, $\beta$-catenin and its downstream genes $c-M y c$ and $c y c l i n D 1$ were significantly decreased after ICA treatment $(\mathrm{p}<0.01)$. Fig. 7 shows that the protein expression level in caspase-3 was significantly increased after ICA treatment $(\mathrm{p}<0.01)$.

Detection of the expression levels of VEGF and MMP-9 via ELISA. The protein expression levels in VEGF and MMP-9 in osteosarcoma cell 143B treated with ICA $(5 \mu \mathrm{M})$ were detected via ELISA. The results are shown in Fig. 8. The protein expression levels in VEGF and MMP-9 after ICA treatment were significantly decreased compared with those in the blank control group $(\mathrm{p}<0.01)$.

\section{Discussion}

Osteosarcoma is a clinically common malignant bone tumor occurring in young individuals, and its main clinical manifestations are progressively aggravated bone pain and local swelling $(14,15)$. The 5-year survival rate of osteosarcoma patients is low, placing great economic and mental burdens on the society and family $(16,17)$. The effective constituents in natural medicine and traditional Chinese medicine have been regarded as the ideal source of antitumor drugs with high efficiency and low toxicity. Previous studies have shown that herb Epimedium has a significant development potential for antitumor drugs. ICA is one of the most studied effective constituents in the studies of herb Epimedium. Li et al (18) studied and found that ICA can cause the death of lung cancer cells in vivo, which has an inhibitory effect on lung cancer cell migration and infiltration. Zhang et al (19) found that ICA can promote the proliferation and differentiation of osteoblasts, which may be realized via the BMP2/Smads/Runx2/Osterix signaling pathways. Hartemayer et al (20) studied and proved that the Wnt/ $\beta$-catenin signaling pathway in chondrocytes can lead to hypertrophy and matrix mineralization of chondrocytes, induce the expressions of MMP-9 and VEGF and promote cell proliferation and migration. In addition, non-steroidal anti-inflammatory drugs can inhibit the proliferation of tumor cells by reducing $\beta$-catenin/TCF transcriptional activity and disturbing the signal transduction of the Wnt pathway (21).

In the present study, the effect of ICA on the proliferation of osteosarcoma cell 143B was investigated and it was found that the proliferation of 143B was significantly inhibited when the concentration of ICA reached $5 \mu \mathrm{M}$. At the same time, the flow cytometry showed that the cell apoptosis after ICA treatment was significantly increased. The aforementioned results indicated that ICA has a good inhibitory effect on the proliferation of osteosarcoma cells with a low onset concentration and high activity. The expression levels of related genes were detected via semi-quantitative PCR, and the results showed that the expression level of $\beta$-catenin was significantly decreased after ICA treatment, while the expression levels of its downstream genes $c$-Myc and cyclin Dl were also significantly decreased. Previous findings have shown that multiple ligands and receptors in the $\mathrm{Wnt} / \beta$-catenin signaling pathway are highly expressed in osteosarcoma cells, suggesting that ICA may inhibit the proliferation of osteosarcoma cells by inhibiting the transcriptional level of $\beta$-catenin (22). Additionally, the protein expression levels in p-GSK $3 \beta$ and $\beta$-catenin were significantly decreased after ICA treatment. During typical Wnt/ $\beta$-catenin signal transduction, the intracellular accumulation of $\beta$-catenin and its transfer to the nucleus were dominated in regulating the signal activity. GSK3 $\beta$ is an important negative regulator in the signal transduction process. Activation of GSK $3 \beta$ can promote the phosphorylation of $\beta$-catenin and eventually lead to the degradation of $\beta$-catenin and inactivation of the $\mathrm{Wnt} / \beta$-catenin signal (23). ICA can increase the catalytic activity of GSK3 $\beta$ by inhibiting the phosphorylation of 9 -serine of GSK3 $\beta$, thus promoting $\beta$-catenin degradation and downregulating the protein level of $\beta$-catenin in cells. It was also found that the expression levels of downstream proteins of $\beta$-catenin, $\mathrm{c}-\mathrm{Myc}$ and cyclin D1, were also inhibited. Therefore, it was inferred that ICA inhibits the $\mathrm{Wnt} / \beta$-catenin signal and the expression of related target proteins in the pathway through inhibition of the phosphorylation of $\mathrm{p}-\mathrm{GSK} 3 \beta$, thus regulating the proliferation and invasion of osteosarcoma cells. At the same time, the expression level of caspase-3 in osteosarcoma cells after ICA treatment was increased, suggesting that ICA can also inhibit the proliferation of osteosarcoma cells by inhibiting their apoptosis.

In conclusion, ICA has a significant effect of inhibiting the proliferation and inducing the apoptosis of osteosarcoma cell $143 \mathrm{~B}$, which may be realized by activating GSK $3 \beta$ and inhibiting the activity of the $\mathrm{Wnt} / \beta$-catenin signaling pathway. However, there were still some shortcomings in this experiment. The inhibitory effect of ICA on osteosarcoma cell proliferation and its mechanism were investigated only through in vitro experiments, and the abovementioned results were not verified in in vivo experiments. In the follow-up studies, the author aims to verify the efficacy of ICA and the role of $\mathrm{Wnt} / \beta$-catenin signaling pathway from in vivo experiments again in the hope to provide new ideas for the efficacious development of ICA and the clinical treatment of osteosarcoma.

\section{Competing interests}

The authors declare that they have no competing interests. 


\section{References}

1. Shimizu T, Kido A, Honoki K, Murata K, Fujii H, Higuchi B, Ishihara T, Takeshita Y, Shima M, Yajima H, et al: A successful reconstruction using a frozen autograft and a pedicled latissimus dorsi flap after a S12345B shoulder girdle resection in a patient with osteosarcoma. J Reconstr Microsurg 28: 155-159, 2012.

2. Qiu Q, Jiang J, Lin L, Cheng S, Xin D, Jiang W, Shen J and Hu Z: Downregulation of RSK2 influences the biological activities of human osteosarcoma cells through inactivating AKT/mTOR signaling pathways. Int J Oncol 48: 2508-2520, 2016.

3. Hurley C, McCarville MB, Shulkin BL, Mao S, Wu J, Navid F, Daw NC, Pappo AS and Bishop MW: Comparison of (18) F-FDG-PET-CT and bone scintigraphy for evaluation of osseous metastases in newly diagnosed and recurrent osteosarcoma. Pediatr Blood Cancer 63: 1381-1386, 2016.

4. Waresijiang N, Sun J, Abuduaini R, Jiang T, Zhou W and Yuan H: The downregulation of miR 125a 5p functions as a tumor suppressor by directly targeting MMP 11 in osteosarcoma. Mol Med Rep 13: 4859-4864, 2016.

5. Ma Q, Zhang Y, Liu T, Jiang K, Wen Y, Fan Q and Qiu X: Hypoxia promotes chemotherapy resistance by down-regulating SKA1 gene expression in human osteosarcoma. Cancer Biol Ther 18: 177-185, 2017.

6. Li H, Yuan Y,Zhang Y,Zhang X, Gao L and Xu R: Icariin inhibits AMPK-dependent autophagy and adipogenesis in adipocytes in vitro and in a model of Graves' orbitopathy. Front Physiol 8: 45, 2017. doi: 10.3389/fphys.2017.00045.

7. Su YS, Fan ZX, Xiao SE, Lin BJ, Miao Y, Hu ZQ and Liu H: Icariin promotes mouse hair follicle growth by increasing insulin-like growth factor 1 expression in dermal papillary cells. Clin Exp Dermatol 42: 287-294, 2017.

8. Xiao HB, Sui GG and Lu XY: Icariin improves eNOS/NO-pathway to prohibit the atherogenesis of apolipoprotein E null mice. Can J Physiol Pharmacol 95: 625-633, 2017.

9. Qian ZQ, Wang YW, Li YL, Li YQ, Ling-Zhu and Yang DL: Icariin prevents hypertension-induced cardiomyocyte apoptosis through the mitochondrial apoptotic pathway. Biomed Pharmacother 88: 823-831, 2017.

10. Zhang Y, Yin L, Zheng N, Zhang L, Liu J, Liang W and Wang Q: Icariin enhances remyelination process after acute demyelination induced by cuprizone exposure. Brain Res Bull 34: 713-725, 2016

11. Zhang S, Feng P, Mo G, Li D, Li Y, Mo L, Yang Z and Liang D: Icariin influences adipogenic differentiation of stem cells affected by osteoblast-osteoclast co-culture and clinical research adipogenic. Biomed Pharmacother 88: 436-442, 2017.

12. Brennecke P1, Arlt MJ, Muff R, Campanile C, Gvozdenovic A, Husmann K, Holzwarth N, Cameroni E, Ehrensperger F, Thelen M, et al: Expression of the chemokine receptor CXCR7 in CXCR4-expressing human 143B osteosarcoma cells enhances lung metastasis of intratibial xenografts in SCID mice. PLoS One 8: e74045, 2013.
13. Mamie Yu, Suresh K. Selvaraj, Liang-Chu MM, Aghajani S, Busse M, Yuan J, Lee G, Peale F, Klijn C, et al: A resource for cell line authentication, annotation and quality control. Nature 520: 307-311, 2015 .

14. Dang H, Wu W, Wang B, Cui C, Niu J, Chen J, Chen Z and Liu Y: CXCL5 plays a promoting role in osteosarcoma cell migration and invasion in autocrine- and paracrine-dependent manners. Oncol Res 25: 177-186, 2017.

15. Zhang S,Liu L,Lv Z,Li Q, Gong W and Wu H: MicroRNA-342-3p inhibits the proliferation, migration, and invasion of osteosarcoma cells by targeting astrocyte-elevated gene-1 (AEG-1). Oncol Res 8: 435-447, 2017.

16. Xu M, Zhang YY, Wang HF and Yang GS: The expression and function of miRNA-106 in pediatric osteosarcoma. Eur Rev Med Pharmacol Sci 21: 715-722, 2017.

17. Rushing CJ, Rogers DE, Spinner SM and Gajzer DC: A case report of heel pain mimicking plantar fasciitis and osteosarcoma: A unique presentation of a Nora's lesion. J Foot Ankle Surg 56: 670-673, 2017.

18. Li W, Wang L, Chu X, Cui H and Bian Y: Icariin combined with human umbilical cord mesenchymal stem cells significantly improve the impaired kidney function in chronic renal failure. Mol Cell Biochem 428: 203-212, 2017.

19. Zhang L, Wang XZ, Li YS, Zhang L and Hao LR: Icariin ameliorates $\operatorname{IgA}$ nephropathy by inhibition of nuclear factor kappa b/Nlrp3 pathway. FEBS Open Bio 7: 54-63, 2016.

20. Hartemayer R, Kuo C and Kent P: Osteosarcoma metastases with direct cardiac invasion: A case report and review of the pediatric literature. J Pediatr Hematol Oncol 39: 188-193, 2017.

21. Sareddy GR, Kesanakurti D, Kirti PB and Babu PP: Nonsteroidalanti-inflammatory drugs diclofenac and celecoxib attenuatesWnt $/ \beta$-catenin/Tcf signaling pathway in human glioblastomacells. Neurochem Res 38: 2313-2322, 2013.

22. Chen G, Wang C, Wang J, Yin S, Gao H, Xiang LU, Liu H, Xiong Y, Wang P, Zhu X, Yang LI and Zhang R: Antiosteoporoticeffect of icariin in ovariectomized rats is mediated via the $\mathrm{Wnt} / \beta$-catenin pathway. Exp Ther Med 12: 279-287, 2016.

23. Li R, Dong T, Hu C, Lu J, Dai J and Liu P: Salinomycinrepressed the epithelial-mesenchymal transition of epithelialovarian cancer cells via downregulating Wnt/ $\beta$-cateninpathway. Onco Targets Ther 28: 328-335, 2017.

This work is licensed under a Creative Commons Attribution-NonCommercial-NoDerivatives 4.0 International (CC BY-NC-ND 4.0) License. 